\title{
Realidad virtual como medio facilitador de actividad física en población en situación de discapacidad*
}

\author{
Iván Darío Pinzón** \\ Jorge Enrique Moreno***
}

Recibido: 2 de febrero del 2020 • Aceptado: 11 de mayo del 2020

\section{Resumen}

La práctica de actividad física regular tiene efectos positivos y protectores en la salud de los sujetos en diferentes poblaciones. En ciertos casos, la aplicación de las tecnologías de la información y la comunicación como la realidad virtual para su praxis se hace necesaria para establecer medios terapéuticos que flexibilizan los espacios de la rehabilitación de las personas. Esta revisión de literatura tiene como objetivo analizar el uso de realidad virtual para facilitar la práctica de actividad física en población en situación de discapacidad. Los resultados incluyeron publicaciones que mencionan los efectos de la realidad virtual en la promoción de actividad física en dicha población. Posterior a la revisión documental, se determinaron tres apartados: 1) qué es realidad virtual, 2) la realidad virtual como método de rehabilitación del movimiento, y 3 ) realidad virtual, discapacidad y actividad física. Se pudo concluir que la aplicación de protocolos de realidad virtual puede promover cambios positivos en el desempeño físico y funcional en población en situación de discapacidad.

Palabras claves: actividad física, ejercicio, realidad virtual.

Artículo de investigación, desarrollado por el grupo de investigación de Ortopedia y Actividad Física de la Fundación Universitaria de Ciencias de la Salud (FUCs), Bogotá, Colombia.

Citar como: Pinzón, I. D. y Moreno, J. E. (2020). Realidad Virtual como medio facilitador de actividad física en población en situación de discapacidad. Revista de Investigación Cuerpo, Cultura y Movimiento, 10(2), 183-205. DoI: https://doi.org/10.15332/2422474x/6232

** Magíster en Ciencias de la Actividad Física y Deporte, Bucaramanga, Colombia. Correo electrónico: ivandpr@hotmail.com, ORCID: 0000-0002-5949-2930

*** Ph. D. en Fisioterapia; director del Programa de Fisioterapia de la Fundación Universitaria de Ciencias de la Salud (FUCS), Bogotá, Colombia. Correo electrónico: jemoreno1@ fucsalud.edu.co, ORCID: 0000-0002-7786-6174 


\title{
Virtual Reality as a Facilitator of Physical Activity in People with Disabilities
}

\begin{abstract}
The practice of regular physical activity has positive and protective effects on the health of subjects in different populations. In certain cases, the use of information and communication technologies such as virtual reality for the practice becomes necessary to establish therapeutic means that make flexible the spaces for the rehabilitation of people. This narrative literature review aims to analyze the use of virtual reality to facilitate the practice of physical activity in people with disabilities. The results include publications that mention the effects of virtual reality in the promotion of physical activity in that population. After the literature review, three sections were determined: 1. What is virtual reality, 2. Virtual reality as a method of movement rehabilitation, and 3. Virtual reality, disability, and physical activity. It was concluded that the application of virtual reality protocols can promote positive changes in physical and functional performance in population with disabilities.
\end{abstract}

Keywords: physical activity, exercise, virtual reality. 


\section{Introducción}

En el mundo, las personas en situación de discapacidad (PSD) permanente se estiman alrededor de un $10 \%$ en la población mundial. Si a esto se le suma que el $15 \%$ de la población presenta una discapacidad transitoria y un $12 \%$ corresponde a adultos mayores con alguna limitación, se llega casi al 40 \% de la población en el mundo con algún impedimento o restricción en sus capacidades (García-Sánchez y Ospina-Rodríguez, 2008). Aunque la discapacidad es un problema de salud pública que se ha incrementado en los últimos años, han ocurrido cambios conceptuales y se han fortalecido las políticas a nivel nacional e internacional. Sin embargo, las PSD experimentan barreras para su inclusión y participación, cuyos principales factores limitantes son de orden físico, político, personal y social que evitan su interacción en escenarios relacionados con la práctica de actividad física (AF) y recreación, las actividades laborales y con el acceso a servicios de salud y educación (Serrano-Ruiz et al., 2013).

Este colectivo es muy heterogéneo en sus etiologías, estadios evolutivos y complejidad de las limitaciones, disfunciones y discapacidades. Las tecnologías de la información y la comunicación (TIC), si bien son una esperanza, también pueden constituir una barrera entre ciudadanos, si no incorporan la accesibilidad a las PSD (García-Bilbao y Rodríguez-Porrero, 2000). A lo largo de la historia, los avances tecnológicos han ofrecido soluciones para mejorar las condiciones de vida y en particular de las PSD: en primer lugar, fueron tecnologías de baja complejidad de tipo mecánico, como las sillas de ruedas y bastones; en una segunda etapa, se incorporó la electrónica a las ayudas técnicas existentes y se crearon sistemas de control de entorno, sistemas braille informatizados, audífonos, entre otros. Gracias a la realidad virtual (Rv), se puede mencionar una tercera generación, en la medida en la tecnología se adapta a las posibilidades de la persona, simplificando el modo de interactuar con las máquinas (Lombardera, 2015). Las TIC permean todos los sectores sociales, permitiendo alcanzar metas que parecían lejanas; sin embargo, las PSD experimentan dificultades para acceder a estas tecnologías y situaciones como ver videos en Internet o escribir un correo electrónico pueden tornarse complejas. Afortunadamente, se han desarrollado herramientas como los sistemas de comandos por voz o sistemas de eye-tracking para la ejecución de estas tareas (Fernández del Pozo, 2016). 
Desde esta óptica, la RV es una de las TIC con mayor auge, constituida por un sistema informático que genera representaciones de la realidad en tiempo real. Los movimientos del mundo real se proyectan en un mundo virtual, permitiendo al usuario experimentar la sensación de estar dentro del espacio ficticio viviendo múltiples emociones (Sunrise Medical, 2018). Basado en esto, y analizando los patrones de movimiento descritos en PSD, se puede desarrollar un sistema que les permita una interacción con el sistema en la ejecución de actividades funcionales y recreativas (Fernández del Pozo, 2016).

Más de 15 años han pasado desde que comenzaran las primeras discusiones acerca de la aplicación de RV en la neuropsicología. Desde entonces, se han desarrollado aplicaciones neuropsicológicas de RV en personas con disfunciones del sistema nervioso central para mejorar las funciones atencionales, amnésicas, ejecutivas o visoespaciales. Los resultados de las investigaciones, referidos especialmente a la precisión alcanzada en la manipulación de los estímulos y a la relevancia y validez de las tareas cognitivas presentadas, fueron dando crédito a las altas expectativas iniciales respecto a los potenciales usos de la RV, y contribuyeron a que esta pasara de considerarse un costoso juguete a una tecnología funcional (Pérez-Salas, 2008).

La promoción de la AF en PSD es un reto que se puede lograr mediante el uso de Rv. Desafortunadamente, la Organización Mundial de la Salud (oms, 2020) reporta que al menos un $60 \%$ de la población mundial no realiza AF necesaria, en parte a la insuficiente participación durante el tiempo de ocio y aumento de comportamientos sedentarios durante las actividades laborales/ domésticas y el uso excesivo de los medios de transporte. Según la Organización Panamericana de la Salud (ops, 2019), casi las tres cuartas partes de la población adulta es sedentaria, siendo las personas de bajo nivel socioeconómico, las mujeres y los adultos mayores las más inactivas durante el tiempo libre; adicionalmente, una proporción considerable de la mortalidad ocasionada por enfermedades crónicas no transmisibles (ECNT) puede atribuirse a los efectos de la inactividad física (Hernández, Gómez y Parra, 2010). El informe Healthy People 2010, presentado por el Centers for Disease Control and Prevention (CDC, 2010) y el President's Council on Physical Fitness and Sports, señaló que las personas inactivas tienen el doble de probabilidades de desarrollar cardiopatías que las personas activas regularmente. Por ello, se reconoce la promoción de AF como estrategia para el mejoramiento de la calidad de vida, en virtud de las situaciones emergentes de salud pública que representa 
el sedentarismo en el ámbito mundial y que se acentúa en el colectivo de PSD (García-Sánchez y Ospina-Rodríguez, 2008).

La mayoría de las investigaciones llevadas a cabo sobre RV se refieren a cómo presentar un mundo simulado en un modo convincente (Bates, 1991). La tecnología adaptativa puede llegar a reducir el impacto de la discapacidad y satisfacer el derecho de la calidad de vida de las personas con necesidades especiales, así como llegar a impactar positivamente la economía después de un importante número de PSD que utilizan como un medio efectivo el teletrabajo. Por otro lado, las mejoras tecnológicas y, sobre todo, la creciente competitividad entre fabricantes hacen que cada día se tengan más en cuenta las necesidades de las personas desde el mismo momento de la concepción de un producto. Pese a que esas necesidades en principio sean las de la población en general, las soluciones adoptadas acaban beneficiando a sectores de población con discapacidad (Koon y De la Vega, 2000).

Por ello, el uso de la RV en un ámbito más educación en la salud pueden conllevar a mejorías en el conocimiento, las habilidades motoras y en el desarrollo de técnicas para resolver problemas complejos en diversas poblaciones, sobre todo en PSD. Desarrollar hábitos saludables y amplificar el saber tecnológico en una sociedad donde cada día se informatizan más aspectos de la vida diaria como la adopción de AF en la vida de los sujetos debe ser piedra angular en las intervenciones de salud pública modernas (Diego-Cordero, FernándezGarcía y Badanta-Romero, 2017). Por eso, este artículo busca revisar el papel de la RV como mediador en la práctica de AF, específicamente en PSD, como estrategia paraa aplicar por parte de los profesionales de la salud.

\section{Metodología}

Se realizó una revisión narrativa de publicaciones relacionadas con el uso de la $\mathrm{RV}$ para promover la práctica de AF en PSD. Se realizó rastreo de información en las bases Pubmed, Medline, Scielo y PEDro mediante los términos MeSH: virtual reality, hándicap, exercise y physical therapy. Los criterios de selección de la información fueron artículos en idioma inglés, portugués y español, entre 2000 y 2018, que mencionan los efectos de la RV sobre la promoción de AF en PSD. Como ruta de búsqueda se siguió la siguiente: virtual reality [Title/ Abstract]) AND handicap [Title/Abstract] AND exercise [Title/Abstract] AND 
physical therapy [Title/Abstract] y tras combinarse con el término booleano OR para evitar duplicidad. Del total de publicaciones elegibles (Pubmed [14], Medline [2], Scielo [21] y PEDro [108]), se excluyeron artículos con deficiente información en la temática o de los cuales no se encontró texto completo. Al final se seleccionaron 60 bibliografías, que incluyeron artículos, referencias impresas, en línea y libros, referenciadas en el presente documento.

\section{Resultados}

\section{¿Qué es realidad virtual?}

El concepto de RV surgió hace varios años; sin embargo, para las personas con poco conocimientos tecnológicos, sigue siendo algo ficticio y desconocido (Jiménez, 2014). La RV es un sistema de computación empleado para crear un mundo artificial donde el usuario cree estar introducido, percibiendo simulaciones creadas por el sistema, tomándolas como si fueran reales y poseer la habilidad de navegar y manipular objetos en el mismo entorno (Manetta y Blade, 1995). Sus características principales son la inmersión (el usuario solo percibe estímulos creados por el entorno virtual), la interacción (el usuario es capaz de interactuar con el entorno virtual en tiempo real) y la imaginación (capaces de concebir realidades que no existen).

La RV puede ser inmersiva (basada en un entorno tridimensional y la realidad física del usuario es reemplazada por el entorno artificial) o no inmersiva (se muestra el entorno en una pantalla). En ambas es posible interactuar a través de periféricos de entrada o de los movimientos corporales. Utiliza tecnologías y elementos para lograr la RV: software de RV, motor de realidad, hardware acorde a la calidad requerida, dispositivos que sumerjan al usuario en el entorno, sistema de renderizado con una tasa de refresco de imagen entre 20 a 30 fotogramas por segundo para dar la sensación de movimiento fluido, sistema de rastreo para mantener la posición ubicando al usuario en el entorno virtual y tecnologías que traduzcan los movimientos físicos reales a movimientos en el entorno artificial (Cano, 2018; Jiménez, 2014).

La RV está relacionada con la ciencia ficción, la simulación y el diseño tridimensional, y sus orígenes se funden con los primeros desarrollos de estas técnicas. Aunque se suele mencionar a Mort Heilig como pionero de la simulación interactiva, el primero en hablar del término RV fue Ivan Southerland 
y, en 1982, Myron Krueger publicó su libro Artificial Reality. Posteriormente, uno de los impulsos más importantes lo dio Willian Gibson en su novela Neuromante, que incluía el concepto ciberespacio. El resultado de las investigaciones militares y aeronáuticas que se desarrollaron en la década de los años 70 , para simular entornos peligrosos y experimentar en ellos sin riesgos, ha dado lugar a la mayor parte de las técnicas que actualmente se utilizan en la RV (Díez, 1995). En la actualidad, los campos de acción de la RV se han extendido a varias ciencias como la salud, donde es muy utilizada en procedimientos e intervenciones terapéuticas.

La realidad virtual como método de rehabilitación del movimiento humano

Existen aplicaciones de RV en el campo de la rehabilitación que incluyen áreas comunes en accidente cerebrovascular (ACV), daño cerebral adquirido, Parkinson, ortopedia, entrenamiento de equilibrio, movilidad en silla de ruedas, actividades funcionales y telerrehabilitación. De estos avances surgen cuatro hallazgos principales: (1) las PSD parecen capaces de aprender dentro de entornos virtuales; (2) los movimientos aprendidos en VR se transfieren a tareas motoras equivalentes del mundo real; (3) se han encontrado ventajas para el entrenamiento de RV en todos los casos; y (4) hasta la fecha, no se ha informado de casos de ciberesmabilidad en los experimentos (Holden, 2005).

La RV se utiliza en distintos ambientes, siendo sus usos más destacados e importantes los siguientes:

- Simulación. Adoptado principalmente por el ejército norteamericano, para realizar el entrenamiento y mejorar habilidades sin arriesgar vidas ante diversas situaciones de combate, es una experiencia totalmente inmersiva que utiliza un Head-Mounted Display, trajes de datos, guantes de datos y armas de Rv.

- Medicina. Es útil para personas con fobias o con estrés postraumático, enfrentándolos ante sus peores miedos. Esta intervención logra una reducción significativa de los síntomas. Otro uso es el control del dolor fantasma en pacientes amputados. Ha demostrado ser de gran ayuda al disminuir o desaparecer estos dolores al crear un entorno virtual en el que el paciente pueda simular mover el miembro amputado. 
- Telepresencia. Área nueva que aprovecha las posibilidades de la RV para permitir que una persona pueda actuar como si estuviese en un lugar, estando realmente en otro. Esto posibilita que un cirujano pueda operar, siendo en realidad un robot el que hace la acción, pero dirigido por el médico a distancia (Jiménez, 2014).

La discapacidad de ciertas personas puede ser amortizada utilizando técnicas de RV; por ejemplo, una persona muda podría hablar en un auditorio heterogéneo utilizando el lenguaje de las manos, pues los movimientos manuales serían enviados a un sintetizador encargado de producir las palabras correspondientes. El desarrollo de los procesadores de señales biológicas permitirá que las señales cerebrales y musculares puedan ser interpretadas, haciendo posible que PSD puedan efectuar acciones sin necesidad de otros medios (Montero-Ayala, 1996). Esta tecnología emergente puede abordar los problemas encontrados en el entrenamiento para manejar un dispositivo de movilidad, entrenar las habilidades de conducción y para mantener la condición física en el tiempo libre (Erren-Wolters, van Dijk, de Kort, IJzerman y Jannink, 2007).

La RV se ha aplicado en el ámbito de la salud de manera exitosa desde la década de los 90, dadas las nuevas oportunidades que ofrecía. Algunas investigaciones han tratado de medir objetivamente la ansiedad y estrés en tiempo real mediante el uso de respuestas fisiológicas como frecuencia cardíaca, respiración, resistencia, actividad periférica de electroencefalograma (EEG) de ondas cerebrales y temperatura de la piel, combinadas con mediciones subjetivas con instrumentos de autorreporte (Wiederhold, Jang, Kim y Wiederhold, 2002). Emmelkampe, Bruynzeel, Drost y Van der mast (2001) realizó un estudio para evaluar la efectividad de la exposición mediante RV versus exposición in vivo en 10 individuos que sufrían de acrofobia (temor patológico a las alturas). Se descubrió que la RV es al menos tan efectiva como la exposición in vivo sobre ansiedad y evitación medida con el cuestionario de acrofobia e incluso más efectivo en el cuestionario de actitud hacia las alturas (Emmelkamp et al., 2001).

Actualmente, se utiliza en neurorrehabilitación, siendo capaz de generar momentos no reales al cerebro y extrapolar al usuario al mundo virtual (Aznar-Díaz, Trujillo-Torres y Romero-Rodríguez, 2018). Específicamente, la terapia se puede proporcionar dentro de un contexto funcional, útil y motivador. Muchas aplicaciones de RV presentan oportunidades para que 
las personas participen en experiencias atractivas y gratificantes. Se utilizan para producir entornos simulados, interactivos y multidimensionales. Las interfaces visuales que incluyen monitores de escritorio y pantallas montadas en la cabeza, interfaces táctiles y dispositivos de seguimiento de movimiento en tiempo real permiten a los usuarios interactuar con imágenes y objetos virtuales a través de múltiples modalidades sensoriales. Las oportunidades para la manipulación de objetos y movimientos corporales proporcionan marcos que son comparables a oportunidades similares en el mundo real (Sveistrup, 2004).

Se ha utilizado la RV en personas con limitaciones cognitivas, parálisis cerebral y síndrome de Down, mejorando la funcionalidad y reintegración vocacional (Chen, Fanchiang y Howard, 2018; Chang, Kang y Huang, 2013; Salem, Gropack, Coffin y Godwin, 2012; Standen, Camm, Battersby, Brown y Harrison, 2010). También existen datos cuantitativos y cualitativos sobre la aplicación de RV para la evaluación y capacitación de usuarios inexpertos en manejo de sillas de ruedas eléctricas, tanto en términos de maniobrabilidad como búsqueda de rutas (Harrison, Derwent, Enticknap, Rose y Attree, 2002).

Leeb (2007) demostró por primera vez que las ondas cerebrales pueden ser utilizadas por un tetrapléjico para controlar su silla de ruedas mediante $\mathrm{RV}$. El sujeto lesionado medular pudo generar explosiones de las oscilaciones $ß$ en el EEG por la imaginación de los movimientos de sus pies paralizados. Estas oscilaciones beta se utilizaron para un control de la interfaz cerebrocomputadora a su propio ritmo (asíncrono) basado en una única grabación bipolar de EEG. El sujeto fue colocado dentro de una calle virtual poblada de avatares y debía ir de avatar a avatar hacia el final de la calle, pero detenerse en cada avatar y hablar con ellos. El participante pudo realizar con éxito esta operación asincrónica con un rendimiento del 90-100 \% (Leeb, Friedman, Muller-Putz, Scherer, Slater y Pfurtscheller, 2007).

En lesiones medulares, la RV ha mostrado ser efectiva para mejorar balance y capacidades de movilidad (An y Park, 2018). Villiger et al. (2017) realizó un estudio con 12 sujetos utilizando una versión móvil en el hogar de un sistema de entrenamiento RV de miembros inferiores. El sistema incluía escenarios de capacitación motivadores y acciones combinadas de observación y ejecución. Las representaciones virtuales de las piernas y los pies se controlaron mediante sensores de movimiento. Los sujetos realizaron entrenamiento en el hogar durante 4 semanas, con 16-20 sesiones de 30-45 min cada una. Las medidas de resultado evaluadas fueron la puntuación motora de la extremidad 
inferior (LEMS), la escala de equilibrio de Berg (BBS), el tiempo acelerado y el avance (TUG). Se eligieron dos puntos de tiempo de evaluación previa al tratamiento para la estabilidad del resultado: 4 semanas antes del tratamiento e inmediatamente antes del tratamiento. En la evaluación inmediatamente después del tratamiento, los sujetos informaron una alta motivación y cambios positivos. Se mostraron mejoras significativas en la fuerza muscular de las extremidades inferiores (LEMS, $p=0.008$ ), el equilibrio ( y la movilidad funcional (TUG, $p=0.007$ ). En la evaluación de seguimiento (es decir, 2-3 meses después del tratamiento), la movilidad funcional (TUG) se mantuvo significativamente mejor $(p=0.005)$ en contraste con las otras medidas de resultado (Villiger et al., 2017).

En el caso del ACv se ha popularizado el uso de RV (Iruthayarajah, McIntyre, Cotoi, Macaluso y Teasell, 2017). Según el grupo canadiense de trabajo Stroke Outcome Research, aunque la rehabilitación estándar (fisioterapia y terapia ocupacional) mejoran la motricidad posterior al ACv, solo hay beneficios modestos hasta la fecha. La comparación de los enfoques de rehabilitación convencionales (técnicas del neurodesarrollo, facilitación neuromuscular propioceptiva o reaprendizaje motor) no ha mostrado diferencias significativas entre los enfoques de tratamiento y los resultados funcionales en ACV. Las nuevas estrategias dirigidas al desarrollo de habilidades motoras, aprovechando los elementos que mejoran la experiencia, ha surgido recientemente, incluidas actividades utilizando tecnología robótica y de RV. Esta última permite interactuar con un entorno multisensorial y recibir retroalimentación sobre el rendimiento, promoviendo los conceptos de neuroplasticidad (es decir, repetición, intensidad y orientación de tareas) y entrenamiento de la extremidad parética (Saposnik y Levin, 2011).

Dada la alta incidencia de lesión cerebral en la población, su rehabilitación es todavía un campo relativamente poco desarrollado. La RV tiene el potencial de ayudar a las técnicas convencionales a abordar las deficiencias, limitaciones en actividades y restricciones en la participación, asociadas con el daño cerebral. El uso de RV en la rehabilitación del daño cerebral se está expandiendo y se convertirá en una parte integral de evaluación cognitiva y rehabilitación en el futuro inmediato para entrenar todas la cualidades y capacidades en PSD (Shih, Chang y Mohua, 2012; Rose, Brooks y Rizzo, 2002).

A medida que las PSD envejecen, las disminuciones progresivas en el estado de salud pueden desafiar los recursos necesarios para mantener la 
independencia funcional y la calidad de vida. Estos desafíos se agravan por factores económicos, efectos secundarios de medicamentos, pérdida de un cónyuge o cuidador y trastornos psicosociales. Si bien la investigación indica que la capacidad motora funcional se puede mejorar, mantener o recuperar mediante la participación constante en un régimen de ejercicio y rehabilitación motor, la adherencia independiente a dicha programación fuera del entorno clínico es notoriamente baja. Esto ha motivado el abordaje de las necesidades de las PSD que envejecen al promover el acceso desde el hogar a sistemas de $\mathrm{RV}$ de bajo costo, diseñados para involucrar y motivar la participación en $\mathrm{AF}$ y rehabilitación. La creación de tales sistemas podría servir para incidir en los procesos sensoriomotores necesarios para maximizar la independencia y la calidad de vida (Rizzo et al., 2011).

\section{Realidad virtual, discapacidad y actividad física}

Los procesos de discapacidad son toda restricción o ausencia de la capacidad de realizar una actividad dentro de parámetros que se consideran normales para el ser humano, lo que genera un situación particular empática desde la interdisciplinariedad (Egea-García y Sarabia-Sánchez, 2001). Es una situación heterogénea donde interactúan las dimensiones físicas, psíquicas y sociales en la que se desarrolla y vive el individuo, e incluye problemas en la función o estructura del cuerpo, pasando por limitaciones en la actividad o en la realización de tareas, hasta la restricción en la participación en las situaciones cotidianas (Padilla-Muñoz, 2010). Se caracteriza por insuficiencias o excesos en el desempeño y comportamiento en una actividad rutinaria, que pueden ser temporales o permanentes, reversibles o irreversibles y progresivos o regresivos, clasificadas en nueve grupos: de la conducta, de la comunicación, del cuidado personal, de la locomoción, de la disposición del cuerpo, de la destreza, de situación, de una determinada aptitud y otras restricciones de la actividad (Cáceres-Rodríguez, 2004; Charpentier y Aboiron, 2000).

La discapacidad es una situación por múltiples causas y casi todas las personas tendrán una discapacidad temporal o permanente en algún momento de sus vidas, y los que sobrevivan y lleguen a la vejez experimentarán cada vez más dificultades de funcionamiento. En cada época se ha enfrentado la cuestión moral y política de encontrar la mejor forma de incluir y apoyar a las PSD. Históricamente se habían tratado con soluciones que las segregaban, 
como las instituciones residenciales y escuelas especiales. Pero en la actualidad la política ha cambiado y se ha optado por la inclusión en la comunidad y en la educación, y las soluciones orientadas al componente médico dan lugar a enfoques que reconocen que la discapacidad se origina tanto en los factores corporales como ambientales (OMS, 2011).

En la última encuesta de discapacidad, autonomía personal y situaciones de dependencia en España, en relación con las actividades que les gustaría hacer en el tiempo libre pero que no pueden por su discapacidad, las PSD eligen el ejercicio físico en primera opción con $23 \%$, antes que viajar (22\%), realizar manualidades y artesanía (12\%) o hacer compras (11\%), panorama que refleja que la AF es un elemento motivador y de inclusión en PSD (Instituto Nacional de Estadística [INE], 2008). En las características de la AF adaptada predominan deportes como baloncesto y atletismo, en contextos competitivos de máximo nivel, principalmente en los Juegos Paralímpicos. La práctica de AF y deporte puede ser un elemento importante de promoción de la independencia, la autoestima y la integración social y laboral de las PSD (Moya-Mata, Ruiz-Sanchis, Ruiz, Pérez y Ros-Ros, 2017). No obstante, esto se ve limitado por los imaginarios sociales acerca de la discapacidad, que aún hoy en día priman en algunas sociedades.

Se ha comprobado que los niveles de AF son muy bajos en todos los grupos etarios en PSD. Hinckson y Curtis (2013) realizaron una revisión sistemática de las medidas de AF en niños con discapacidad intelectual (DI); la búsqueda identificó 78 artículos eligibles para su revisión completa y 30 cumplieron los criterios de inclusión. A pesar de las diferencias en el diseño del estudio y la calidad metodológica, hubo acuerdo entre los estudios que los niños con DI eran significativamente menos activos en comparación con los niños sin discapacidades. La negativa a usar instrumentos, las limitaciones de movimiento en niños con identificación y la colocación de dispositivos fueron problemas comunes (Hinckson y Curtis, 2013).

En adolescentes y adultos jóvenes con síndrome de Down (SD), se investigaron los efectos de un programa de entrenamiento de resistencia progresiva (PRP). Participaron 68 jóvenes (30 mujeres, 38 hombres; edad media $17.9 \pm 2.6$ años) con discapacidad intelectual leve a moderada, asignados aleatoriamente a un programa de PRP $(n=34)$ o un grupo social $(n=34)$. Los participantes en el grupo PRP entrenaron dos veces/semana durante diez semanas en un gimnasio guiados por un fisioterapeuta. El grupo social completó un programa de diez 
semanas de actividades sociales con un mentor estudiantil una vez/semana durante 90 minutos. El rendimiento laboral, la fuerza muscular y los niveles de AF fueron evaluados en las semanas 0,11 y 24 . El grupo PRP aumentó su fuerza de miembros superiores e inferiores en la semana 11 en comparación con el grupo control, pero solo su fuerza muscular de miembros inferiores en la semana 24. Hubo una diferencia significativa en los niveles de AF en el grupo PRP en la semana 24 (Shields, Taylor, Wee, Wollersheim, O'Shea y Fernhall, 2013).

Hilgenkamp, Reis, Wijck y Evenhuis (2012) realizaron un estudio para medir los niveles de AF en 1050 adultos mayores ( $\geq 50$ años) con DI. Allí se midieron, con un podómetro, los pasos/día de los participantes. En gran parte debido a limitaciones físicas ( $\mathrm{n}=103)$, velocidad de caminata $<3.2 \mathrm{~km} / \mathrm{h}$ ( $\mathrm{n}=252)$, comprensión limitada o falta de cooperación $(\mathrm{n}=233)$, solo 257 del grupo pudieron participar en mediciones válidas con podómetros $\mathrm{y}$, de estos, solo el $16.7 \%$ (IC $95 \%$ 12.2-21.3) cumplió con la directriz de 10000 pasos/día, el $36.2 \%$ (IC $95 \%$ 30.3-42.1) tomó 7500 pasos/día o más, y el $39 \%$ (95\% IC 32.6-44.5) fue sedentario (< 5000 pasos/día). Debido a que la muestra medida era la parte más funcional de la muestra total, es probable que este resultado sea una sobreestimación considerable de los niveles reales de AF; sin embargo, se muestra que los niveles de AF son extremadamente bajos en adultos mayores con DI (Hilgenkamp et al., 2012).

Uno de los sistemas corporales más comprometido con el sedentarismo es el cardiovascular/pulmonar en PSD. La aptitud cardiorrespiratoria es la capacidad de los sistemas circulatorio, respiratorio y muscular para suministrar oxígeno durante la AF sostenida. Se han encontrado bajos niveles de aptitud cardiorrespiratoria en DI, lo que los pone en mayor riesgo de enfermedades cardiovasculares y mortalidad por todas las causas. Los niveles de aptitud cardiorrespiratoria de niños y adolescentes con DI son bajos, y disminuyen aún más con el aumento de la edad. Además, las mujeres tienen niveles de aptitud cardiorrespiratoria más bajos que los hombres. La inactividad física y la incompetencia cronotrópica tienen más probabilidades de contribuir a los bajos niveles de aptitud cardiorrespiratoria en PSD (Oppewal, Hilgenkamp, Wijck y Evenhuis, 2013).

Hay poca información disponible sobre los cambios de la aptitud física y los programas de intervención entre PSD. Wu (2010) investigó la efectividad de los programas de forma física saludable en PSD intelectual 
institucionalizadas. 146 participantes con edad de 19-67 años fueron reclutados en el estudio. Recopiló información sobre la condición de discapacidad (tipo y nivel), altura, peso, IMC y estado físico (incluye prueba de sentado y alcance en forma de $\mathrm{V}$, sentadillas en $30 \mathrm{seg}$ y $60 \mathrm{seg}$, y carrera) en el inicio y seis meses después de la intervención. Los resultados mostraron disminuciones estadísticas en el peso del individuo, el IMC, la categoría de IMC y la mejoría positiva en la prueba de sentarse y alcanzar en forma de $\mathrm{V}$, en las pruebas de 30 seg y 60 seg después de intervenciones de seis meses. Los resultados también mostraron que el grupo de nivel de discapacidad leve tiene la mayor efectividad en el programa de acondicionamiento físico saludable para disminuir el peso corporal (Wu et al., 2010).

Pese a este panorama, la Rv ha mostrado efectos positivos en la modificación de los niveles de AF. En un estudio cuasiexperimental se comparó el efecto de la terapia ocupacional estándar (TOE) y la realidad virtual usando la tecnología de juegos Wii (RVWii) para mejorar las funciones sensoriomotoras en niños con SD. 105 niños fueron asignados aleatoriamente a la intervención con TOE o RVWii, mientras que otros 50 sirvieron como controles. Todos fueron evaluados con medidas de las funciones sensoriomotoras. En la post-intervención, los grupos de tratamiento superaron significativamente al grupo de control en todas las medidas y los participantes en el grupo RVWii mostraron mayor cambio pre-post en la competencia motora, las habilidades de integración visual y el funcionamiento integrador sensorial (Wuang, Chiang, Su y Wang, 2011).

En sujetos con discapacidades intelectuales y del desarrollo (DID), Lotan Yalon-Chamovitz y Weis (2009) evaluaron la efectividad de un programa de ejercicios basado en VR para mejorar la condición física. Un grupo de investigación $(\mathrm{n}=30$; edad $=52.3 \pm 5.8$ años; nivel de DID moderado) se comparó por edad, nivel de DID y habilidades funcionales con un grupo de control $(\mathrm{n}=30$, edad $=54.3 \pm 5.4$ años). Se llevó a cabo un programa de acondicionamiento físico de seis semanas, dos sesiones de 30 minutos/semana, que incluye ejercicios similares a los de un juego proporcionados por el sistema Sony PlayStation II EyeToy VR. Los cambios en la aptitud física se monitorearon mediante el índice de gasto de energía (EEI), Cooper modificado y el Índice de latido total del corazón (тнві). Se demostraron mejoras significativas $(p<0.05)$ en la aptitud física para el grupo intervenido en comparación con el grupo control para la prueba de Cooper modificado y el THBI, pero no para la prueba de EEI (Lotan et al., 2009). 
Dębska, Polechoński, Mynarski y Polechoński (2019) evaluaron el disfrute y la intensidad del ejercicio físico mientras se practica la AF en RV inmersiva (RVI) utilizando dispositivos de entrenamiento innovadores (cinta de correr omnidireccional Omni y simulador de vuelo Icaros Pro). El estudio también contiene los resultados de una investigación subjetiva sobre la utilidad de tal forma de AF en opinión de los usuarios. En total, 61 adultos (10 mujeres y 51 hombres) participaron en el estudio. Para evaluar el nivel de disfrute (EL) se utilizó la subescala de Interés/Disfrute del Inventario de Motivación Intrínseca (IMI). La intensidad del ejercicio se evaluó durante las sesiones de 10 minutos de videojuegos activos (AVG) en RVI en función de la frecuencia cardíaca. El nivel de disfrute promedio durante el ejercicio físico en RVI en los dispositivos de entrenamiento probados fue alto (Omni 5.74 puntos, Icaros 5.60 puntos en una escala Likert de 1-7) y difirió significativamente a favor de la AF en Omni. En la opinión de la mayoría de los participantes, los AVG en RVI en los dispositivos probados constituyen una forma suficientemente útil de AF para satisfacer las necesidades de las actividades de tiempo libre, e incluso pueden reemplazar algunas formas de esfuerzo físico realizadas de una manera clásica. La intensidad de la AF durante los juegos en dispositivos de entrenamiento estuvo en el nivel recomendado para beneficios de salud para el $80.55 \%$ (Omni) y el 50.77 \% (Icaros Pro) de su duración (Dębska et al., 2019).

Basado en esto, se debe desmitificar la visión de las PSD cuando practica AF o algún deporte. Según Schell y Rodríguez (2001), los deportistas con discapacidad no suelen reflejarse de manera frecuente en los medios de comunicación y, cuando lo hacen, suele ser por temas controversiales más que por sus logros deportivos. En muchos casos las PSD son objeto de representaciones sociales negativas, siendo consideradas con menos valor y un cuerpo con discapacidad es percibido como débil, frágil y dependiente (Pappous, Marcellini, De Léséleuc y Rio-Valle, 2009). Además, la silla de ruedas se estigmatiza como emblema de la discapacidad en los imaginarios y, si se le asocia el sexo, hay un fuerte sesgo en la representación femenina, al ser estereotipadas como frágiles, dependientes, pasivas e incapaces de controlar sus sentimientos (De Léséleuc, Pappous y Marcellini 2009; Pappous et al., 2009; Thomas y Smith, 2003), demostrando que la mujer con discapacidad sufre doble discriminación (Hargreaves y Hardin, 2009). 


\section{Conclusiones}

Son múltiples y variados los alcances de la RV que proporciona un medio poderoso para aumentar los niveles de interacción ambiental en un entorno altamente controlado y de manera estructurada en PSD. La característica vital de la RV es la interactividad y dentro del mundo virtual el usuario debe adaptarse en términos de procesos mentales y comportamiento. La aplicación de RV a pacientes con daño cerebral ofrece una forma única y poderosa de aumentar la cantidad y calidad de la interacción directa con el entorno y de la reducción de las deficiencias cognitivas y conductuales. Por lo tanto, el entorno de RV ofrece una oportunidad única y segura para la intervención física funcional (Johnson, Rushton y Shaw, 1996).

Los profesionales de la salud y el ejercicio están llamados a incorporar las TIC en su quehacer profesional, y en especial la RV, que proporciona seguridad y reintegración social en el colectivo de PSD, con el fin de mantener la condición física saludable, evitar el detrimento de la salud y mejorar la calidad de vida de estos sujetos. Estas tecnologías se pueden adquirir a bajo costo de manera comercial, e indirectamente apoyarán los niveles de movimiento en ambientes seguros, dando diversión y diversificando la manera de concebir la AF. Según la oms (2020), y desde una mirada de salud pública, las tecnologías médicas son objetivos importantes del sistema internacional, en particular de la labor que realiza el sistema de las Naciones Unidas en su conjunto, de la que cabe destacar los Objetivos de Desarrollo del Milenio, cuyo objetivo fundacional es alcanzar para todos los pueblos el grado más alto posible de salud (ops, 2019; OMs, OMPI y OMC, 2013).

Según el artículo "La nueva tendencia para ponerte en forma: usar realidad virtual para hacer ejercicios en otro mundo" de la BBC, incluso para aquellos que no sienten particular interés o atracción por la actividad física, como los millones de gamers que en el mundo prefieren pasar horas sedentarias frente a un videojuego antes que estirar los músculos, la RV es una excelente opción a ser aprovechada y que puede extrapolarse a una amplia variedad de sujetos; la RV "claramente fue mejor que los programas de noticias sin sonido que ponen en los gimnasios. Pero no puedo decir que sea algo que me vaya a atraer a largo plazo, aunque debo reconocer que tampoco lo hace ir al gimnasio" (párr. 26), comentó Zoe Kleinman enviada de la BBC al Consumer Electronics Show (CEs) y quien probó la RV (BBC News, 2018). 
En general, la RV es una gran herramienta para propender cambios positivos en el desempeño físico y funcional en PSD. Todos estos cambios deben estar alineados con las recomendaciones de AF propuestas por la OMS y ampliamente difundidas por estamentos como el American College of Sport Medicine, dirigidas a las distintas poblaciones acorde a los grupos de edad; sin embargo, aún hay poco campo trasegado en el área de la discapacidad. Desde este punto, se convierte en una oportunidad de análisis, intervención e investigación que puede servir para mejorar el cuerpo del conocimiento de la AF en discapacidad, lo cual repercute positivamente en la expectativa de vida de esta población.

\section{Conflictos de interés}

No se declaran conflictos de intereses.

\section{Agradecimientos}

Los autores agradecen el apoyo recibido por el programa de Fisioterapia de la FUCS para la elaboración del escrito.

\section{Referencias}

An, C. M. y Park, Y.H. (2018). The effects of semi-immersive virtual reality therapy on standing balance and upright mobility function in individuals with chronic incomplete spinal cord injury: A preliminary study. Journal of Spinal Cord Medicine, 41(2), 223-229. DoI: http://doi.org/10.1080/10790268.2017.1369 217

Aznar-Díaz, I., Trujillo-Torres, J. M. y Romero-Rodríguez, J. M. (2018). Estudio bibliométrico sobre la realidad virtual aplicada a la neurorrehabilitación y su influencia en la literatura científica. Revista Cubana de Información en Ciencias de la Salud, 29(2), 1-10. Recuperado de http://scielo.sld.cu/scielo. php?script=sci_arttext\&pid=S2307-21132018000200009\&lng=es\&nrm=iso \&tlng=es 
Bates, J. (1991). Virtual Reality, Art, and Entertainment. Pittsburgh, PA: School of Computer Science and College of Fine Arts Carnegie Mellon University.

BBC News. (2018). La nueva tendencia para ponerte en forma: usar realidad virtual para hacer ejercicios en otro mundo. Recuperado de https://www.bbc.com/ mundo/deportes-42608056

Cáceres-Rodríguez, C. (2004). Revisión teórica sobre el concepto de discapacidad. Una revisión de las propuestas de la oms. Revista Electrónica de Audiología , 2, 1-4.

Centers for Disease Control and Prevention (cdc). (2010). Healthy People 2010: Physical Activity and Fitness. Recueperado de https://www.cdc.gov/nchs/data/ hpdata2010/hp2010_final_review_focus_area_22.pdf

Chang, Y. J., Kang, Y. S. y Huang, P. C. (2013). An augmented reality (ar)-based vocational task prompting system for people with cognitive impairments. Research in Developmental Disabilities, 34(10), 3049-3056. DoI: http://doi. org/10.1016/j.ridd.2013.06.026

Charpentier, P. y Aboiron, H. (2000). Classification internationale des handicaps. Encyclopédie Médico-Chirurgicale. Paris: Editions Scientifiques et Médicales Elsevier.

Chen, Y., Fanchiang, H. C. y Howard, A. (2018). Effectiveness of Virtual Reality in Children with Cerebral Palsy: A Systematic Review and Meta-Analysis of Randomized Controlled Trials. Physical Therapy, 98(1), 63-77. DoI: http:// doi.org/10.1093/ptj/pzx107

De Léséleuc, E, Pappous, A. y Marcellini, A. (2009). La cobertura mediática de las mujeres deportistas con discapacidad: análisis de la prensa diaria de cuatro países europeos durante los Juegos Paralímpicos de Sydney 2000. Apunts. Educación Física y Deportes, 97, 80-88. Recuperado de https://www.raco.cat/ index.php/ApuntsEFD/article/view/300101

Dębska, M., Polechoński, J., Mynarski, A. y Polechoński, P. (2019). Enjoyment and Intensity of Physical Activity in Immersive Virtual Reality Performed on Innovative Training Devices in Compliance with Recommendations for Health. International Journal of Environmental Research and Public Health, 16(19): 3673-3685. DoI: http://doi.org/10.3390/ijerph16193673

Diego-Cordero, R., Fernández-García, E., Badanta-Romero, B. (2017). Uso de las tic para fomentar estilos de vida saludables en niños/as y adolescentes: el caso del sobrepeso. Revista Española de Comunicación en Salud, 8(1), 79-91. Recuperado de https://e-revistas.uc3m.es/index.php/RECS/article/view/3607 
Díez, L. A. G. (1995). Evolución de la tecnología militar y” su impacto” en España. Cuadernos de estrategia, 75, 83-114. Recuperado de https://dialnet. unirioja.es/descarga/articulo/2779486.pdf

Egea-García, C. y Sarabia-Sánchez, A. (2001). Clasificaciones de la oms sobre discapacidad. Boletín del RPD, 50, [en línea]. Recuperado de http://www.cedd.net/ docs/ficheros/200405120002 _ 24 _O.pdf

Emmelkamp, P. M. G., Bruynzeel, M., Drost, L. y Van der mast, C. A. P. G. (2001). Virtual Reality Treatment in Acrophobia: A Comparison with Exposure in Vivo. Cyberpsychology \& Behavior, 4(3), 335-339. DoI: http://doi. org/10.1089/109493101300210222

Erren-Wolters, C. V., van Dijk, H., de Kort, A. C., IJzerman, M. J. y Jannink, M. J. (2007). Virtual reality for mobility devices: training applications and clinical results: a review. International Journal of Rehabilitation Research, 30(2), 9196. doi: http://doi.org/10.1097/MRR.0b013e32813a2e00

Fernández del Pozo, C. (2016). Realidad Virtual para personas que tienen discapacidad motora, (Tesis de grado). Universidad Autónoma de Madrid, España. Recuperado de http://hdl.handle.net/10486/673436

García-Bilbao, A. y Rodríguez-Porrero, C. (2000). Nuevas tecnologías y personas con discapacidad. Intervención Psicosocial, 9(3), 283-296. Recuperado de https://dialnet.unirioja.es/servlet/articulo?codigo=2009250

García-Sánchez, L. V. y Ospina-Rodríguez, J. (2008). Imaginarios de las personas en situación de discapacidad en torno a la actividad física. Revista de Ciencias de la Salud, 6(2), 51-63. Recuperado de http://repositoriocdpd.net:8080/ handle/123456789/120

Gibson, W. (2014). Neuromante. Milán: Edizioni Mondadori.

Hargreaves, J. y Hardin, B. (2009). Women wheelchair athletes: competing against media stereotypes. Disability Studies Quarterly, 29(2), 1095-1096. Recuperado de http://dsq-sds.org/article/view/920.

Harrison, A., Derwent, G., Enticknap, A., Rose, F. D. y Attree, E. A. (2002). The role of virtual reality technology in the assessment and training of inexperienced powered wheelchair users. Journal Disability and Rehabilitation, 24(1112),599-606. DoI: http://doi.org/10.1080/09638280110111360

Hernández, A., Gómez, L. F. y Parra, D. (2010). Ambientes urbanos y actividad física en adultos mayores: Relevancia del tema para América Latina. Revista de Salud Pública, 12(2), 327-335. DoI: https://doi.org/10.1590/ S0124-00642010000200016 
Hilgenkamp, T. I. M., Reis, D., Wijck, R. V. y Evenhuis, H. M. (2012). Physical activity levels in older adults with intellectual disabilities are extremely low. Research in Developmental Disabilities, 33(2), 477-483. DoI: http://doi. org/10.1016/j.ridd.2011.10.011

Hinckson, E. A. y Curtis, A. (2013). Measuring physical activity in children and youth living with intellectual disabilities: A systematic review. Research in Developmental Disabilities, 34(1), 72-86. DoI: http://doi.org/10.1016/j. ridd.2012.07.022

Holden, M. K. (2005). Virtual Environments for Motor Rehabilitation: Review. Cyberpsychology \& Behavior, 8(3), 187-211. DoI: https://doi.org/10.1089/ cpb.2005.8.187

Instituto Nacional de Estadística (INE). (2008). Encuesta de Discapacidad, Autonomía personal y situaciones de Dependencia. Recuperado de http:// www.ine.es/prensa/np524.pdf

Iruthayarajah, J., McIntyre, A., Cotoi, A., Macaluso, S. y Teasell, R. (2017). The use of virtual reality for balance among individuals with chronic stroke: a systematic review and meta-analysis. Top Stroke Rehabilitation, 24(1), 68-79. DoI: http://doi.org/10.1080/10749357.2016.1192361

Jiménez, R. (2014). Realidad virtual, su presente y futuro. Recuperado de https:// jeuazarru.com/wp-content/uploads/2014/10/Realidad-Virtual-2014.pdf

Johnson, D. A., Rushton, S. y Shaw, J. (1996). Virtual reality enriched environments, physical exercise and neuropsychological rehabilitation. Proc. 1st Euro. Conf. Disability, Virtual Reality of Assoc (pp. 247-151). Maidenhead, uk: University of Reading. Recuperado de https://www.researchgate.net/profile/PM_Sharkey/ publication/260854455_Proceedings_of_the_1st_European_Conference_on_ Disability_Virtual_Reality_and_Associated_Technologies_ECDVRAT_1996/ links/00b7d532833de149ff000000.pdf\#page $=259$.

Koon, R. A. y De la Vega, M. E. (2000). El impacto tecnológico en las personas con discapacidad. Ponencia peresentada en el Congreso CIIEE2000 Córdoba. Recuperado de http://www.repositoriocdpd.net:8080/bitstream/ handle/123456789/363/Pon_KoonRA_ImpactoTecnologicoPersonas_2000. pdf?sequence $=1$

Krueger, M. W. (1991). Artificial reality ii. Reading: Addison-Wesley.

Leeb, R., Friedman, D., Muller-Putz, G.R., Scherer, R., Slater, M., Pfurtscheller, G. (2007). Self-Paced (Asynchronous) BCI Control of a Wheelchair in Virtual Environments: A Case Study with a Tetraplegic. Computational Intelligence and Neuroscience, 2007, 79642. DoI: http://doi.org/10.1155/2007/79642 
Lombardera, L. (2015). Trabajar en la era digital: tecnología y competencias para la transformación digital. Córdoba: Editorial Almuzara.

Lotan, M., Yalon-Chamovitz, S. y Weis, P. L. (2009). Improving physical fitness of individuals with intellectual and developmental disability through a Virtual Reality Intervention Program. Research in Developmental Disabilities, 30(2), 229-239. DoI: http://doi.org/10.1016/j.ridd.2008.03.005

Manetta, C. y Blade, R. (1995). Glossary of vr terminology. The International Journal of Virtual Reality, 1(2), 35-39. https://doi.org/10.20870/IJVR.1995.1.2.2604

Montero-Ayala, R. (1996). Realidad Virtual. Autores científico-técnicos y académicos ACTA, 01, 51-59. Recuperado de https://docplayer.es/6361233-Realidadvirtual-que-es-la-realidad-virtual-ramon-montero-ayala.html

Moya-Mata, I., Ruiz-Sanchis, L., Ruiz, J. M., Pérez, P. M. y Ros-Ros, C. (2017). La representación de la discapacidad en las imágenes de los libros de texto de Educación Física: ¿inclusión o exclusión? RETOS. Nuevas Tendencias en Educación Física, Deporte y Recreación, 32, 88-95. Recuperado de https:// www.redalyc.org/pdf/3457/345751100018.pdf

Organización Mundial de la Salud (OMS). (2020). Estrategia mundial sobre régimen alimentario, actividad física y salud. Recuperado de https://www.who.int/ dietphysicalactivity/factsheet_inactivity/es/.

Organización Mundial de la Salud (OMS). (2011). Informe mundial sobre la discapacidad. Recuperado de http://www1.paho.org/arg/images/Gallery/Informe_spa. pdf

Organización Mundial de la Salud (OMS), Organización Mundial de la Propiedad Intelectual (OMPI) y Organización Mundial del Comercio (OMC). (2013). Promover el acceso a las tecnologías médicas y la innovación Intersecciones entre la salud pública, la propiedad intelectual y el comercio. Recuperado de https://apps.who.int/iris/bitstream/handle/10665/276351/A71_12-sp.pdf.

Organización Panamericana de la Salud (OPS). (2019). Plan de acción mundial sobre Actividad Física 2018-2030. Más personas activas para un mundo sano. ops.

Oppewal, A., Hilgenkamp, T. I. M., Wijck, R. V., Evenhuis, H. M. (2013). Cardiorespiratory fitness in individuals with intellectual disabilities-A review. Research in Developmental Disabilities, 34(10), 3301-3316. DoI: http://doi. org/10.1016/j.ridd.2013.07.005

Padilla-Muñoz, A. (2010). Discapacidad: contexto, concepto y modelos. International Law: Revista Colombiana de Derecho Internacional, 16, 381414. Recuperado de https://www.redalyc.org/pdf/824/82420041012.pdf 
Pappous, A., Marcellini, A., De Léséleuc, E., Rio-Valle, S., Quintana, F. C., García, M. P. y Muñoz, A. (2009). La representación mediática del deporte adaptado a la discapacidad en los medios de comunicación. Ágora para la Educación Física y el Deporte, 9, 31-42.

Pérez-Salas, C. (2008). Realidad virtual: un aporte real para la evaluación y el tratamiento de personas con discapacidad intelectual. Terapia Psicológica, 26(2), 253-262. DoI: http://doi.org/10.4067/S0718-48082008000200011

Rizzo A, Requejo, P., Winstein, C. J., Lange, B., Ragusa, G., Merians, A., ... Alsen, M. (2011). Virtual reality applications for addressing the needs of those aging with disability. Studies in Health Technology and Informatics, 163, 510-516. DOI: http://doi.org/10.3233/978-1-60750-706-2-510

Rose, F. D., Brooks, B. M. y Rizzo, A. A. (2002). Virtual Reality in Brain Damage Rehabilitation: Review. Cyberpsychology \& Behavior, 8(3), 241-271. DOI: https://doi.org/10.1089/cpb.2005.8.241

Salem, Y., Gropack, S. J., Coffin, D. y Godwin, E. M. (2012). Effectiveness of a lowcost virtual reality system for children with developmental delay: a preliminary randomised single-blind controlled trial. Physiotherapy, 98(3), 189-195. DoI: http://doi.org/10.1016/j.physio.2012.06.003

Saposnik, G. y Levin, M. (2011). Virtual Reality in Stroke Rehabilitation A MetaAnalysis and Implications for Clinicians. Stroke, 42(5), 1380-1386. DoI: http:// doi.org/10.1161/STROKEAHA.110.605451

Schell, L. A. y Rodríguez, S. (2001). Subverting bodies/ambivalent representations: Media analysis of Paralympian, Hope Lewellen. Sociology of Sports Journal, 18, 127-135. DOI: https://doi.org/10.1123/ssj.18.1.127

Serrano-Ruiz, C. P., Ramírez-Ramírez, C., Abril-Miranda, J. P., Ramón-Camargo, L. V., Guerra-Urquijo, L. Y. y Clavijo-González, N. (2013). Barreras contextuales para la participación de las personas con discapacidad física. Salud UIS, 45(1),41-51. Recuperado de https://revistas.uis.edu.co/index.php/ revistasaluduis/article/view/3299.

Shields, N., Taylor, N.F., Wee, E., Wollersheim, D., O'Shea, S. y Fernhall, B. (2013). A community-based strength training programme increases muscle strength and physical activity in young people with Down syndrome: A randomised controlled trial. Research in Developmental Disabilities, 34(12), 4385-4394. doi: http://doi.org/10.1016/j.ridd.2013.09.022

Shih, C. H., Chang, M. L. y Mohua, Z. (2012). A three-dimensional object orientation detector assisting people with developmental disabilities to control their environmental stimulation through simple occupational activities with 
a Nintendo Wii Remote Controller. Research in Developmental Disabilities, 33(2), 484-489. DoI: http://doi.org/10.1016/j.ridd.2011.10.012

Standen, P. J., Camm, C., Battersby, S., Brown, D. J. y Harrison, M. (2010). An evaluation of the Wii Nunchuk as an alternative assistive device for people with intellectual and physical disabilities using switch controlled software. Computers \& Education, 56(1), 2-10. DoI: http://doi.org/10.1016/j.compedu.2010.06.003

Sunrise Medical. (2018). Realidad virtual y discapacidad física: la posibilidad de romper cualquier barrera. [Blog] Publicado 16 Mayo 2018 [en línea] [citado 2019 Dic 20] Recuperado de https://www.sunrisemedical.es/blog/ realidad-virtual-discapacidad/

Sveistrup, H. (2004). Motor rehabilitation using virtual reality. Journal of Neuro Engineering and Rehabilitation, 1(10), 1-8. DoI: http://doi. org/10.1186/1743-0003-1-10

Thomas, N. y Smith, A. (2003). Preoccupied with able-bodiedness? An analysis of the British media coverage of the 2000 Paralympic Games. Adapted Physical Activity Quarterly, 20(2), 166-181. DoI: https://doi.org/10.1123/apaq.20.2.166

Villiger, .M, Liviero, J., Awai, L., Stoop, R., Pyk, P., ... Bolliger, M. (2017). HomeBased Virtual Reality-Augmented Training Improves Lower Limb Muscle Strength, Balance, and Functional Mobility following Chronic Incomplete Spinal Cord Injury. Frontiers in Neurology, 8, 635-643. DoI: http://doi. org/10.3389/fneur.2017.00635

Wiederhold, B. K., Jang, D. P., Kim, S. I. y Wiederhold, M. D. (2002). Physiological Monitoring as an Objective Tool in Virtual Reality Therapy. Cyberpsychology \& Behavior, 5(1),77-82. Recuperado de https://doi. org/10.1089/109493102753685908

Wu, C. L., Lin, J. D., Hu, J., Yen, C. F., Yen, C. T., Chou, Y. L. y Wu, P. H. (2010). The effectiveness of healthy physical fitness programs on people with intellectual disabilities living in a disability institution: Six-month short-term effect. Research in Developmental Disabilities, 31(3), 713-717. Dor: http://doi. org/10.1016/j.ridd.2010.01.013

Wuang, Y. P., Chiang, C. S., Su, C. Y. y Wang, C. C. (2011). Effectiveness of virtual reality using Wii gaming technology in children with Down syndrome. Research in Developmental Disabilities, 32(1), 312-321. DoI: http://doi. org/10.1016/j.ridd.2010.10.002 
